\title{
Stellar Orbits in Doubly-Barred Galaxies
}

\author{
H. Hasan ${ }^{1}$ \\ Astrophysics Division, NASA Headquarters, Washington D.C. 20546 \\ and \\ Jet Propulsion Laboratory, California Institute of Technology, \\ 4800 Oak Grove Dr., Pasadena CA 91109
}

\section{Introduction}

The question of bars within bars has been reviewed by Friedli and Martinet (1993), who have also performed N-body simulations to produce nested bars. They propose that if a system of embedded bars is effective in transporting gas to the galactic center (Shlosman et al. 1989), then it is perhaps a step in the secular evolution of barred galaxies. In order to pursue this interesting proposition, and also because observational evidence for the existence of secondary bars is mounting (e.g. Buta \& Crocker 1993; Shaw et al. 1993, Wozniak et al. 1995), it is important to understand the stellar kinematics in such systems.

As a first step in this direction, we compute stellar orbits in a two dimensional potential representing the galaxy and examine surfaces of section (s.o.s.) in the galactic plane. We consider the secondary bar to rotate at the same pattern speed and to be (a) parallel and (b) perpendicular to the primary bar. In each case we study the effect of increasing the mass of the secondary bar and of changing its width. We find that increasing the mass of the secondary bar causes the appearance of resonances, particularly for the perpendicular bar, when there is a rich resonance structure in the surface of section. The effect of the resonances is, of course, to destabilize the B (or $x_{1}$ ) orbits which support the bar. The effect of the inner bar in this sense is similar to that of a spherical central mass, the growth of which causes bar destruction (Hasan \& Norman 1990). A similar effect is seen when we keep the mass of the secondary bar constant, but make it more concentrated by reducing its width (cf. Hasan et al. 1993 for the spherical central mass case). An interesting difference we observe in the surfaces of section of the case of a spherical central mass and an inner bar is, that in the latter case increasing the central mass produces resonance structures on the retrograde side as well. In the former case, retrograde orbits were not strongly affected by a growth of a central mass (Hasan et al. 1993).

We propose to follow up this study by computing periodic orbits in a three dimensional model potential and also for the more interesting case of two bars inclined at an arbitrary angle and rotating at different pattern speeds.

\footnotetext{
${ }^{1}$ On leave from: Space Telescope Science Institute, 3700 San Martin Dr., Baltimore, MD 21218
} 


\section{Stellar Orbits, Surfaces of Section and ILR}

Stellar orbits are computed in a galactic potential, the disk component of which is modeled by a Plummer sphere and the primary and secondary bars as prolate Ferrers bars. For normalization purposes it is found convenient to fix the primary bar semi-major axis $a_{1}$ at $9 \mathrm{kpc}$, and the total mass $M_{T}=4.67 \times 10^{10} M_{\odot}$ (Hasan $\&$ Norman 1990). For all cases studied this normalization results in a pattern speed $\Omega_{p} \sim 15 \mathrm{~km} \mathrm{~s}^{-1} \mathrm{kpc}^{-1}$ so that $R_{c r} / a_{1}=1$, where $R_{c r}$ is the corotation radius. The parameters fixed for all calculations are: the primary bar mass, $M_{b_{1}} / M_{T}=0.3$, its semi-minor axis, $b_{1}=4.05 \mathrm{kpc}$, secondary bar major axis, $a_{2}=1.8 \mathrm{kpc}$ and the length scale of the central component, $A_{c} / a_{1}=0.5$. The Jacobi constant was fixed so that a star could reach a maximum distance corresponding to the edge of the minor axis of the primary bar.

At first, the minor axis of the secondary bar is fixed to $0.45 a_{2}=0.81$ $\mathrm{kpc}$ and surfaces of section computed for several secondary bar masses and axis ratios, keeping the secondary bar (1) parallel and (2) perpendicular to the primary bar. Both bars are assumed to rotate at the same pattern speed. A similar analysis is repeated keeping the secondary bar mass fixed at $0.05 M_{T}$ and varying the secondary bar axial ratio.

\subsection{Variation of Secondary Bar Mass}

An examination of the surfaces of section for parallel bars (case I) and perpendicular bars (case II) shows that in the presence of a small secondary bar $\left(M_{b_{2}} / M_{T}=0.05\right)$ the direct orbits which support the bar (the $\mathrm{B}$ or $x_{1}$ orbits) fill almost all the space available to direct orbits. As the mass of the secondary bar increases the interaction of the primary and secondary bars causes the appearance of resonances, represented by islands, both on the direct and retrograde sides. Further, the region of stochasticity increases and the phase space occupied by $\mathrm{B}$ orbits shrinks, thus causing a weakening of the primary bar. Nevertheless, the primary bar is not destroyed until the secondary bar is quite massive $(\sim 20 \%$ of the total mass).

For secondary bar masses $\sim 0.07 M_{T}$ a minor ("banana") orbit family appears for case II. With increasing secondary bar mass it slowly disappears and the invariant curves representing $B$ orbits change from an oval shape to a more rectangular to a diamond shape, indicating that the influence of the secondary bar is to give the galaxy a boxier shape. The orbits are still elongated along the primary bar, but develop complicated looped shapes as higher order resonances set in. Stochasticity along both bars increases and eventually both bars will be destroyed. For case I, the banana orbits do not appear till $M_{b_{2}} / M_{T}=0.11$, and strengthen with increasing secondary bar mass. The significance of this family, as discussed in our earlier analyses of central masses (Hasan et al. 1993), is that these orbits take stars out of the galactic plane and could help populate the bulge.

\subsection{Variation of Secondary Bar Axial Ratio}

The problem was re-examined in a complementary scheme, when the secondary bar mass was fixed at $0.05 M_{T}$ and gradually concentrated within the secondary bar by changing the axial ratio $b_{2} / a_{2}=0.45,0.35,0.25$. All other parameters 
were held constant. An examination of surfaces of section shows that a more concentrated inner bar causes more stochasticity than a thicker, less dense bar. In case II, for $b_{2} / a_{2}=0.25$, some of the $\mathrm{B}$ orbits are converted to A orbits, which manifest themselves in the s.o.s. as islands along the edges of the s.o.s. along the zero velocity curve.

Role of resonances in bar destruction Physical insight may be gained by looking at the frequency curves for each case. Plotting the quantity, $\Omega-\kappa / 2$, where $\Omega$ and $\kappa$ represent the "circular" and "epicyclic" frequency respectively of an equivalent circular potential, we see that for all cases considered, the pattern speed, $\Omega_{p}$, lies below the peak in the curves $\Omega-\kappa / 2$, indicating the presence of Inner Lindblad Resonances (ILRs). For the case of the parallel bars there are two ILRs corresponding to the two bars. Between the potential center and the first ILR, and the second ILR and corotation, orbits will be parallel and support bars, while in the region between the two ILRs, orbits will be antialigned to the bar. As the secondary bar mass increases in mass, the outer ILR moves closer to the corotation radius, thus reducing the phase space available to $\mathrm{B}$ orbits supporting the primary bar, which eventually gets destroyed. At the same time, the inner ILR moves closer to the potential center, reducing the phase space available to the secondary bar and causing its destruction.

For the case of the perpendicular bars, only the outer ILR is present. The orbits close to the potential center are antialigned, thus supporting the secondary bar. With increase in mass of the secondary bar, as in case I, the outer ILR moves outwards thus weakening the primary bar.

Acknowledgments. I would like to acknowledge technical support from the Space Telescope Science Institute, Baltimore. The research described in this paper was carried out by the Jet Propulsion Laboratory, California Institute of Technology, under a contract with the National Aeronautics and Space Administration.

\section{References}

Buta, R. \& Crocker, D. A. 1993, AJ, 105, 1344

Friedli, D. \& Martinet, L. 1993, A\&A, 277, 27

Hasan, H. \& Norman, C. 1990, ApJ, 361, 69

Hasan, H., Pfenniger, D., \& Norman, C. 1993, ApJ, 409, 91

Shlosman, I., Frank, J., \& Begelman, M. C. 1989, Nature 338, 45

Shaw, M. A., Combes, F., Axon, D. J., \& Wright, G. G. 1993, A\&A, 273, 31

Wozniak, H., Friedli, D., Martinet, L., Martin, P., \& Bratschi, P. 1995, A\&AS, 111,115 